\title{
Outage and Diversity of Linear Receivers in Flat-Fading MIMO Channels
}

\author{
Ahmadreza Hedayat Member, IEEE, and Aria Nosratinia Senior Member, IEEE
}

\begin{abstract}
This correspondence studies linear receivers for MIMO channels under frequency-nonselective (flat) quasi-static Rayleigh fading. The outage probability and diversity gain of MMSE and Zero Forcing (ZF) receivers are investigated. It is found that contrary to intuition, MMSE and zero-forcing receivers may not perform similarly at high SNR. Assuming $M$ transmit and $N$ receive antennas, the zero-forcing receiver always has diversity $N-M+1$, unlike the MMSE receiver, whose behavior can vary. Under separate spatial encoding, where data from each transmit antenna is separately encoded, MMSE is no better than $\mathrm{ZF}$ in terms of diversity. But for joint spatial encoding systems, where an encoded stream is sent from all the antennas, the MMSE receiver achieves diversity $M N$ at low spectral efficiencies but has diversity only $M-N+1$ at high spectral efficiencies.
\end{abstract}

\section{INTRODUCTION}

The fading wireless MIMO channel is characterized by a mixing (interference) of the signals arriving from multiple transmitter antennas. Sometimes multiple transmit antennas are only used to increase reliability, e.g. orthogonal space-time codes, thus the signals from multiple antennas are tightly structured and the interference can be undone at the receiver. However, transmit signals do not always have as much structure (e.g. in the case of spatial multiplexing) thus more elaborate methods may be needed to remove the spatial interference [1], [2]. For example, nulling-and-cancelling detectors [1], [2], [3] are capable of providing optimal detection.

Linear receivers, even though suboptimal, are much simpler and therefore useful for many applications. In this correspondence, we study linear MIMO receivers under quasistatic flat fading. We calculate the diversity of linear receivers via their outage probabilities. Since the outage probability is closely related to the frame error rate, this also provides a tangible measure of the performance of realistic systems. A summary of our results is as follows: For a MIMO system consisting of $M$ transmit and $N$ receive antennas, under flat Rayleigh fading, zero-forcing receivers achieve diversity order $M-N+1$ under all cases studied. MMSE receivers achieve the same diversity for transmission strategies that do not allow combined coding of data streams, e.g. horizontal spatial encoding. However, for coding strategies that allow joint encoding of data streams, e.g. D-BLAST, a more interesting scenario emerges. In such systems, for low spectral efficiencies MMSE receivers can achieve the full diversity of $M N$, while

A. Hedayat is with Navini Networks, Richardson, TX, USA Email: hedayat@ieee.org. A. Nosratinia is with the Dept. of Electrical Engineering, The University of Texas at Dallas, Richardson, TX, USA Email:aria@utdallas.edu. This work was presented in part at ICASSP 2005. for high spectral efficiencies only a diversity of $M+N-1$ is possible. For intermediate values of $R$, diversities in between the two extremes are observed.

\section{LINEAR RECEIVERS}

The input-output system model for flat fading MIMO channel with $M$ transmit and $N \geq M$ receive antennas is $\mathbf{r}=$ $\mathbf{H c}+\mathbf{n}$, where $\mathbf{c}$ is the $M \times 1$ transmitted vector, $\mathbf{n} \in \mathcal{C}^{N \times 1}$ is the Gaussian noise vector, and $\mathbf{r}$ is the $N \times 1$ received vector at a given time instant. Throughout this paper, we assume $\mathbf{H}$ has independent and identically distributed complex Gaussian entries, i.e. $\mathbf{H} \in \mathcal{C}^{N \times M}$.

We consider linear receivers and evaluate the outage probability of a flat fading MIMO channel followed by a ZF or MMSE receiver, assuming the channel is perfectly known to the receiver. The ZF receiver is $\mathbf{F}_{\mathrm{ZF}}=\left(\mathbf{H}^{H} \mathbf{H}\right)^{-1} \mathbf{H}^{H}$, which transforms the received signal to

$$
\hat{\mathbf{r}}=\mathbf{F}_{\mathrm{ZF}} \mathbf{r}=\mathbf{c}+\left(\mathbf{H}^{H} \mathbf{H}\right)^{-1} \mathbf{H}^{H} \mathbf{n} .
$$

The MMSE receiver is $\mathbf{F}_{\text {MMSE }}=\left(\mathbf{H}^{H} \mathbf{H}+\rho^{-1} \mathbf{I}\right)^{-1} \mathbf{H}^{H}$, where $\rho$ is the received SNR.

Since the symbols are detected individually, the SINR of the individual symbols determines the performance. The detection noise of ZF receiver, $\tilde{\mathbf{n}} \triangleq\left(\mathbf{H}^{H} \mathbf{H}\right)^{-1} \mathbf{H}^{H} \mathbf{n}$, is a complex Gaussian vector with zero-mean and covariance matrix $\mathbf{R}_{\tilde{\mathbf{n}}}=$ $\sigma_{n}^{2}\left(\mathbf{H}^{H} \mathbf{H}\right)^{-1}$. The $k$ th diagonal element of $\mathbf{R}_{\tilde{\mathbf{n}}}$ is given by:

$$
\mathbf{R}_{\tilde{\mathbf{n}}}(k, k)=\sigma_{n}^{2}\left(\mathbf{H}^{H} \mathbf{H}\right)_{k}^{-1}=\sigma_{n}^{2} \frac{\operatorname{det}\left(\hat{\mathbf{H}}^{H} \hat{\mathbf{H}}\right)}{\operatorname{det}\left(\mathbf{H}^{H} \mathbf{H}\right)},
$$

where $(\mathbf{M})_{k}^{-1}$ represents the $k$ th diagonal element of the inverse of $\mathbf{M}$, and $\hat{\mathbf{H}}$ is obtained by removing the $k$ th row of $\mathbf{H}$. The associated SINR is $\gamma_{k}=\mathcal{E}_{x} / \mathbf{R}_{\tilde{\mathbf{n}}}(k, k)$, which can be shown to be a chi-square random variable with $2(N-M+1)$ degrees of freedom [4], [5]. The CDF of $Y \sim \chi_{2(N-M+1)}$, with variance 0.5 for the participating Gaussian random variables, is:

$$
F_{Y}(y)=1-e^{-y} \sum_{i=1}^{N-M+1} \frac{y^{i-1}}{(i-1) !} .
$$

The SINR of the $k$ th symbol of MMSE detector is determined by noise and residual interference

$\gamma_{k}=\mathbf{h}_{k}^{H}\left(\hat{\mathbf{H}}_{k} \hat{\mathbf{H}}_{k}^{H}+\rho^{-1} \mathbf{I}\right)^{-1} \mathbf{h}_{k}=\frac{1}{\left(\mathbf{I}+\rho \mathbf{H}^{H} \mathbf{H}\right)_{k}^{-1}}-($ (B)

where $\mathbf{h}_{k}$ is the $k$ th column of $\mathbf{H}$. Removing this column from $\mathbf{H}$ gives $\hat{\mathbf{H}}_{k} \in \mathcal{C}^{N \times(M-1)}[6]$. 
Equation (3) shows that $\gamma_{k}$ is a quadratic form whose statistics has been derived in [7] as follows. Considering the random matrix $\hat{\mathbf{H}} \in \mathcal{C}^{N \times(M-1)}$ and the random vector $\mathbf{h} \in \mathcal{C}^{N}$, the quadratic form $Y=\mathbf{h}^{H}\left(\hat{\mathbf{H}} \hat{\mathbf{H}}^{H}+\rho^{-1} \mathbf{I}\right)^{-1} \mathbf{h}$ has the $\mathrm{CDF}$

$$
F_{Y}(y)=1-\exp \left(-\frac{y}{\rho}\right) \sum_{n=1}^{N} \frac{A_{n}(y)}{(n-1) !}\left(\frac{y}{\rho}\right)^{n-1},
$$

where the auxiliary functions $A_{n}(y)$ are given by

$$
A_{n}(y)=\left\{\begin{array}{ll}
1 & N \geq M+n-1 \\
\frac{1+\sum_{i=1}^{N-n} C_{i} y^{i}}{(1+y)^{M-1}} & N<M+n-1 .
\end{array},\right.
$$

and $C_{i}$ is the coefficient of $y^{i}$ in $(1+y)^{M-1}$ [7]. In general, the SINR of the output symbols of the MMSE receiver are correlated, unlike those of the zero-forcing receiver.

\section{Outage Probability in Separate Spatial ENCODING}

In separate spatial encoding, the data stream is demultiplexed to several sub-streams, one for each transmit antenna. Furthermore, the resulting streams are not jointly encoded, to achieve easier decoding. Horizontally encoded V-BLAST is a prominent example of this strategy. In this scenario, if any of the data streams is in outage, the entire system is in outage. Hence, the outage event $\mathcal{O}$ occurs when any of the subchannels cannot support the rate that is assigned to it. In our analysis, we consider equal rate for the sub-channels, however, it is also possible to have a non-uniform rate assignment.

After linear transformation, the mutual information between the elements of $\hat{\mathbf{r}}$ and the transmitted data vector $\mathbf{c}$ is $\mathcal{I}\left(c_{k} ; \hat{r}_{k}\right)=\log \left(1+\gamma_{k}\right)$. Assume the target rate is $R$, and let $L \triangleq N-M$. According to (2) and (4), the statistics of $\gamma_{k}$ is invariant to $k$. Thus, the outage probability $\operatorname{Pr}(\mathcal{O})$ is :

$$
\begin{aligned}
\operatorname{Pr}(\mathcal{O}) & =1-\operatorname{Pr}\left(\bigcap_{k=1}^{M}\left\{\mathcal{I}\left(c_{k} ; \hat{r}_{k}\right) \geq \frac{R}{M}\right\}\right) \\
& =1-\left(\operatorname{Pr}\left(\mathcal{I}\left(c_{k} ; \hat{r}_{k}\right) \geq \frac{R}{M}\right)\right)^{M} \\
& \approx M \operatorname{Pr}\left(\mathcal{I}\left(c_{k} ; \hat{r}_{k}\right)<\frac{R}{M}\right),
\end{aligned}
$$

where (6) is accurate when sub-channel outage probabilities are small. In the above, we have assumed that sub-channel outage events are independent, which is valid for ZF. For MMSE receivers the sub-channel outage events are not strictly independent, but the approximation (used only in this section) makes the analysis tractable and does not affect diversity. Simulations show that the approximation has been properly used. Alternatively, one may consider only the outage event of a single sub-channel, which is an approximation that is accurate enough for diversity calculation.

Using the CDF of $\chi_{2(N-M+1)}$ in the evaluation of (6) gives the outage probability for the $\mathrm{ZF}$ receiver, which is

$$
\begin{aligned}
\operatorname{Pr}(\mathcal{O}) & \approx M F_{Y}\left(2^{R / M}-1\right) \\
& \stackrel{M}{=} \frac{M\left(2^{R / M}-1\right)^{L+1}}{(L+1) !} \rho^{-(L+1)},
\end{aligned}
$$

where $\stackrel{\circ}{=}$ denotes equivalence in the limit as $\rho \rightarrow \infty$. Thus the ZF diversity order is $L+1$. Substituting the distribution (4) in (6), the MMSE outage probability is calculated:

$$
\begin{aligned}
& \operatorname{Pr}(\mathcal{O}) \approx M F_{Y}\left(2^{\frac{R}{M}}-1\right) \\
& \left.\stackrel{{ }^{L+1}}{=} \frac{y^{M-1}}{(L+1) !} \cdot \rho^{-(L+1)}\right|_{y=2 \frac{R}{M}-1}
\end{aligned}
$$

which shows that MMSE diversity order is also $L+1$. However, the ZF and MMSE outage probabilities are not exactly the same. The ratio of (7) to (8) is:

$$
\frac{\operatorname{Pr}(\mathcal{O})_{\mathrm{ZF}}}{\operatorname{Pr}(\mathcal{O})_{\mathrm{MMSE}}}=\left.\frac{(1+y)^{M-1}}{y^{M-1}}\right|_{y=2^{\frac{R}{M}}-1}=\left(\frac{2^{\frac{R}{M}}}{2^{\frac{R}{M}}-1}\right)^{M-1} \text {. }
$$

Note that the ratio of outage probabilities in (9) remains fixed regardless of SNR and it only depends on the relative target rate $\frac{R}{M}$. When $\frac{R}{M}$ is small the outage probability of ZF becomes larger than that of MMSE. The ratio (9) approaches one when $\frac{R}{M}$ is large (see Section V).

Generalization of the above results to non-uniform rate assignment is straightforward. Uniform and non-uniform rate assignment have the same diversity, even though they have different outage probability performance.

\section{Outage Probability in Joint Spatial Encoding}

In joint spatial encoding, the data stream is encoded and then demultiplexed into sub-streams, each going to one antenna (e.g. D-BLAST). Thus, each data symbol can contribute to signals of all the transmit antennas. The receiver is in outage when the aggregate mutual information of all the sub-channels fails to support the target rate.

The mutual information between the elements of the linearly transformed receive signal, $\hat{\mathbf{r}}$ and the transmitted data vector c is $\mathcal{I}\left(c_{k} ; \hat{r}_{k}\right)=\log \left(1+\gamma_{k}\right)$. Assuming the target rate is $R$, the probability of the outage event $\mathcal{O}$ is

$$
\begin{aligned}
\operatorname{Pr}(\mathcal{O}) & =\operatorname{Pr}\left(\sum_{k=1}^{M} \log \left(1+\gamma_{k}\right)<R\right) \\
& =\operatorname{Pr}\left(\prod_{k=1}^{M}\left(1+\gamma_{k}\right)<2^{R}\right) .
\end{aligned}
$$

Theorem 1: Consider a flat MIMO channel with $M$ transmit and $N \geq M$ receive antennas, and joint spatial encoding. Under perfect channel state information available to the receiver, the outage probability of $\mathrm{ZF}$ receivers decays with order of $N-M+1$.

Proof: See the Appendix.

Thus, we observe that the ZF diversity is the same for separate and joint spatial encoding.

To obtain the MMSE outage probability, we substitute the SINR from (3) in (11), which gives:

$$
\operatorname{Pr}(\mathcal{O})=\operatorname{Pr}\left(\prod_{k=1}^{M}\left(\mathbf{I}+\rho \mathbf{H}^{H} \mathbf{H}\right)_{k}^{-1}>2^{-R}\right) .
$$

The dependence on the diagonal elements of the random matrix $\left(\mathbf{I}+\rho \mathbf{H}^{H} \mathbf{H}\right)^{-1}$ makes further analysis intractable. 
Therefore, we proceed to provide an upper bound to this probability. Rewriting the sum mutual information as in (10), we have

$$
\begin{aligned}
-\sum_{k=1}^{M} \mathcal{I}\left(c_{k} ; \hat{r}_{k}\right) & =\sum_{k=1}^{M} \log \left(\left(\mathbf{I}+\rho \mathbf{H}^{H} \mathbf{H}\right)_{k}^{-1}\right) \\
& \leq M \log \left(\sum_{k=1}^{M} \frac{1}{M}\left(\mathbf{I}+\rho \mathbf{H}^{H} \mathbf{H}\right)_{k}^{-1}\right) \\
& =M \log \left(\frac{1}{M} \operatorname{tr}\left(\left(\mathbf{I}+\rho \mathbf{H}^{H} \mathbf{H}\right)^{-1}\right)\right) \\
& =M \log \left(\frac{1}{M} \sum_{k=1}^{M} \frac{1}{1+\rho \lambda_{k}}\right),
\end{aligned}
$$

where (13) is due to Jensen's inequality, and $\lambda_{k}$ 's are the eigenvalues of the Wishart matrix $\mathbf{H}^{H} \mathbf{H}$. Substituting (14) into (10) gives:

$$
\operatorname{Pr}(\mathcal{O}) \leq \operatorname{Pr}\left(\sum_{k=1}^{M} \frac{1}{1+\rho \lambda_{k}} \geq M 2^{-\frac{R}{M}}\right) .
$$

Though (15) is an upper bound of the outage probability, in Section $\mathrm{V}$, through simulation, we show that it is a tight upper bound in low and high spectral efficiency. Assuming $N \geq M$, the joint PDF of the eigenvalues of $\mathbf{H}^{H} \mathbf{H}, \lambda_{k}$ 's, $\lambda_{1} \leq \lambda_{2} \leq$ $\cdots \leq \lambda_{M}$, is

$$
f_{\boldsymbol{\Lambda}}(\boldsymbol{\lambda})=K_{M, N} \prod_{i=1}^{M} \lambda_{i}^{N-M} \prod_{i<j}\left(\lambda_{i}-\lambda_{j}\right)^{2} \exp \left(-\sum_{i} \lambda_{i}\right),
$$

where $K_{M, N}$ is a normalizing constant [8].

The evaluation of (15) for a specific outage rate $R$ is rather difficult, due to the shape of the outage region. However, one can calculate the bound for small and large values of $R$ where the the outage region can be approximated by regions with simpler shapes.

For a MIMO channel with $M=2$ and $N \geq 2$, the bound (15) is

$$
\operatorname{Pr}(\mathcal{O}) \leq \operatorname{Pr}\left(\frac{1}{1+\rho \lambda_{1}}+\frac{1}{1+\rho \lambda_{2}} \geq 2^{1-\frac{R}{2}}\right) .
$$

For convenience define

$$
S\left(\lambda_{1}, \lambda_{2}\right) \triangleq \frac{1}{1+\rho \lambda_{1}}+\frac{1}{1+\rho \lambda_{2}}
$$

and also define the set

$$
\mathcal{A} \triangleq\left\{\left(\lambda_{1}, \lambda_{2}\right): \frac{1}{1+\rho \lambda_{1}}+\frac{1}{1+\rho \lambda_{2}} \geq 2^{1-\frac{R}{2}}\right\} .
$$

Then the right hand side of $(17)$ is $\operatorname{Pr}(\mathcal{A})$. Exact calculation of $\operatorname{Pr}(\mathcal{A})$ is not easy, thus we show its asymptotic behavior by bounding it from below and above.

Let $0 \leq R<2$. If $\lambda_{1}=0$ outage occurs only when $\lambda_{2} \leq$ $c_{2} \triangleq \frac{2-b}{\rho(b-1)}$, where $b \triangleq 2^{1-\frac{R}{2}}$. Because the curve $S\left(\lambda_{1}, \lambda_{2}\right)=$ $b$ is convex, the region $\mathcal{A}$ is contained in the isosceles right triangle with the base $\lambda_{1}+\lambda_{2}=c_{2}$ and the two sides $\lambda_{1}=0$ and $\lambda_{2}=0$, and integral over the triangle is always larger than $\operatorname{Pr}(\mathcal{A})$.
We now build another triangle that is contained by $\mathcal{A}$. Using the symmetry of $S\left(\lambda_{1}, \lambda_{2}\right)$, it is not difficult to calculate that an isosceles triangle with base $\lambda_{1}+\lambda_{2}=\tilde{c}_{2}$, where $\tilde{c}_{2}=\frac{(2-b)}{b \rho}$, is contained in $\mathcal{A}$ and integration over this triangle is always smaller than $\operatorname{Pr}(\mathcal{A})$.

Finally, we show that probability integrals over the two triangles behave the same asymptotically, thus completing a sandwich argument. To do so, consider the integral over any such isosceles triangle with parameter $c$ :

$$
\begin{aligned}
K_{2, N} & \int_{0}^{c} e^{-\lambda_{1}} \lambda_{1}^{N-2} \int_{0}^{c-\lambda_{1}} \lambda_{2}^{N-2}\left(\lambda_{1}-\lambda_{2}\right)^{2} e^{-\lambda_{2}} d \lambda_{2} d \lambda_{1} \\
& =2 K_{2, N}(N-1) !(N-2) !\left(1-e^{-c} \sum_{k=1}^{2 N-1} \frac{c^{k}}{k !}\right) \stackrel{\circ}{=} \rho^{-2 N}
\end{aligned}
$$

where $c$ could be $c_{2}$ or $\tilde{c}_{2}$. Since $\operatorname{Pr}(\mathcal{A})$ is bounded above and below by values that have diversity- $2 N$, it must have diversity $2 N$. Now recall that $\operatorname{Pr}(\mathcal{O}) \leq \operatorname{Pr}(\mathcal{A})$ therefore we have established that outage has diversity no less than $2 N$. Considering that $2 N$ is also the maximum achievable diversity order, we conclude that outage has exactly diversity order $2 N$. This concludes the arguments for small spectral efficiencies.

Now we consider high spectral efficiencies, namely $R>2$ and $0 \leq b<1$. In this case, $\lambda_{2}$ can drive the system to outage regardless of the value of $\lambda_{1}$ (and vice versa). For instance, let $\lambda_{1} \rightarrow \infty$, as long as $\lambda_{2} \leq d_{2}=\frac{1-b}{\rho b}$, outage occurs. Thus, the outage region has a strip along the $\lambda_{1}$ axis for large enough $\lambda_{1}$, and likewise along $\lambda_{2}$. In fact, the set of strips defined as $0 \leq \lambda_{2}, 0 \leq \lambda_{1} \leq d_{2}$ and $0 \leq \lambda_{1}, 0 \leq \lambda_{2} \leq d_{2}$ is contained in $\mathcal{A}$. Since $S\left(\lambda_{1}, \lambda_{2}\right)=b$ is convex, it is possible to find $\tilde{d}_{2}$, which is proportional to $\rho^{-1}$ but $\tilde{d}_{2}>d_{2}$, such that $\mathcal{A}$ contains the strips $0 \leq \lambda_{2}, 0 \leq \lambda_{1} \leq \tilde{d}_{2}$ and $0 \leq \lambda_{1}, 0 \leq \lambda_{2} \leq \tilde{d}_{2}$. The probability of the above sets can be characterized using the following expression:

$$
\begin{aligned}
2 K_{2, N} \int_{0}^{d} e^{-\lambda_{1}} \lambda_{1}^{N-2} \int_{0}^{\infty} & \lambda_{2}^{N-2}\left(\lambda_{1}-\lambda_{2}\right)^{2} e^{-\lambda_{2}} d \lambda_{2} d \lambda_{1} \\
& \stackrel{\circ}{=} \rho^{-(N-1)},
\end{aligned}
$$

where $d$ could be $d_{2}$ or $\tilde{d}_{2}$. Therefore (19) indicates that the upper bound (17) has the diversity $N-1=L+1$, where $L=$ $N-M$. In the calculation of (19), the intersection of the two orthogonal strips is calculated twice, but the intersection has a probability that decays with $\rho^{-2(N-1)}$ and does not affect the asymptotic behavior of (19).

The outage bounds developed above show the surprising fact that MMSE receivers can achieve the same diversity as the ML receiver for small values of $R$ in joint spatial encoding. However, for large values of $R$ the diversity performance of MMSE and ZF is the same. Hence, for MMSE the diversity varies from the diversity of an unconstrained receiver to that of $\mathrm{ZF}$, depending of the target rate $R$. Comparing to the results from Section III for separate spatial encoders, the MMSE receiver has different diversity in joint spatial encoding architecture, except for large outage rate $R$.

The previous results of the case $M=2, N \geq 2$ can be extended to arbitrary values of $M$ and $N \geq M$. We state the general result in the following theorem. 


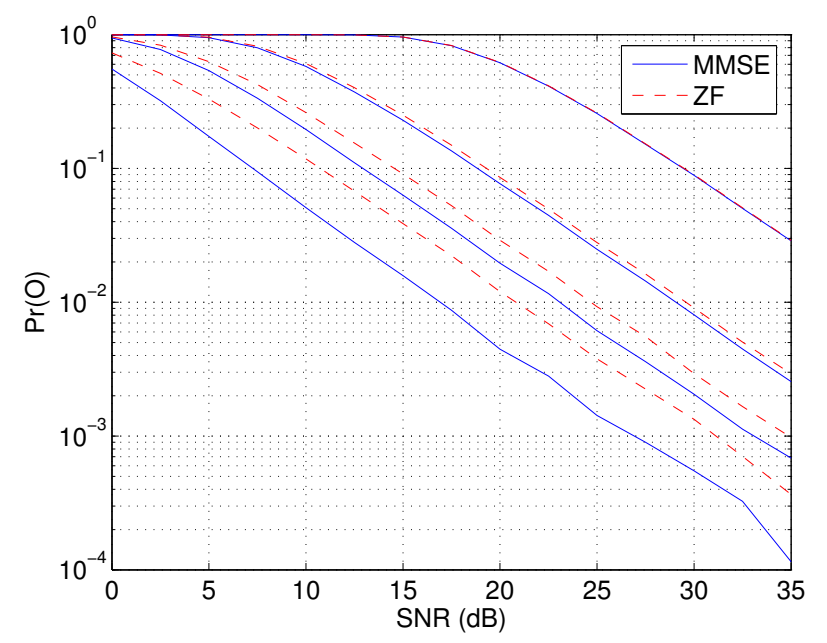

Fig. 1. Outage probability of linear receivers, $M=N=2$. The pairs of solid and dashed lines, from left, correspond to MMSE and ZF for rates $R=1,2,4,10 \mathrm{bits} / \mathrm{sec} / \mathrm{Hz}$

Theorem 2: Consider a flat MIMO channel with $M$ transmit and $N \geq M$ receive antennas, and joint spatial encoding. Under perfect channel state information available to the receiver, the upper bound (15) on the outage probability of MMSE receivers decays with order of $M N$ at low spectral efficiency, i.e. $R<M \log \left(\frac{M}{M-1}\right)$, resulting in the diversity order of $M N$ for the outage probability. At high spectral efficiency $R>M \log M$, (15) decays with the order of $N-M+1$.

Proof: See the Appendix.

\section{Simulation Results}

We consider a MIMO system with two antennas in transmit and receive sides: $M=N=2$. The outage probability of the linear receivers in the separate architecture is shown in Figure 1. The target rate is $R=1,2,4,10 \mathrm{bits} / \mathrm{sec} / \mathrm{Hz}$. As expected, both linear detectors show diversity order of one, regardless of the target rate. For higher values of $R$ the difference of $\mathrm{ZF}$ and MMSE performance is negligible. But, for lower values of $R$, MMSE performs better than $\mathrm{ZF}$ for all SNR. The dependency of the relative performance of these receivers on the target rate $R$ is in agreement with (9). In high SNR, the ratio of the outage probabilities remains fixed.

Figure 2 shows the outage probability of the unconstrained receiver and linear receivers in a joint spatial encoding architecture. The unconstrained receiver has the full diversity of the channel. The ZF receiver has diversity one as expected from the analysis in Section IV. The diversity order of ZF remains unchanged regardless of the target rate $R$. Surprisingly, MMSE diversity depends on $R$ : in lower values of $R$ the diversity order is very close to that of the unconstrained receiver, and in higher values of $R$ its diversity becomes the same as ZF diversity. These results are in agreement with the analysis in Section IV.

Figure 2 also shows the outage probability of the MMSE receiver and the upper bound (15). The bound is tight at either low or high values of $R$. Though the bound is loose in the intermediate values of $R$, it does predict diversity order varying with $R$.

Figure 3 presents similar results for a flat fading MIMO channel with $M=N=2$ and correlated transmit antennas with correlation factor $\rho_{t}=0.5$. Outage probabilities are slightly higher than the uncorrelated case, however, the behavior of outage probabilities are the same. Figure 3 also shows the results for uncorrelated MIMO channel with $M=N=4$.

\section{CONCLUSION}

We present new results on the performance of linear receivers for the removal of spatial interference in MIMO Rayleigh flat fading channels, and calculate their diversity order. Our analytical and experimental results show that MMSE receivers have outage probability with varying decaying slope: it may decay as fast as the outage probability of unconstrained receiver, with the full order of $M N$, or as slowly as that of $\mathrm{ZF}$ receivers, with the order of $N-M+1$, depending on the spectral efficiency.

The authors gratefully acknowledge comments from Dr. Naofal Al-Dhahir.

\section{APPENDIX}

Proof of Theorem 1: The SINR of the sub-channels under $\mathrm{ZF}$ are independent chi-square random variables with degrees $2(N-M+1)$. Let $Y_{k} \sim \chi_{2(N-M+1)}, k=1, \cdots, M$. The outage probability of $\mathrm{ZF}$ is given by the $\mathrm{CDF}$ of the random variable

$$
\prod_{k=1}^{M}\left(1+Y_{k}\right)=1+\sum_{k=1}^{M} Y_{k}+\cdots+\prod_{k=1}^{M} Y_{k} .
$$

Among the components of the above random variable, the last term, which is the product of $Y_{k}$ 's, determines the diversity order since it is a chi-square with the lowest degree. In the following, through recursion, we show that $Y_{1} \cdot Y_{2} \cdots Y_{M}$ has diversity order $L+1$. Let us start by $Z \triangleq Y_{1} \cdot Y_{2}$. The PDF of $Z$ is

$$
f_{Z}(z)=\frac{2}{((L-1) !)^{2}} z^{L} K_{0}(2 \sqrt{z}),
$$

where $K_{0}(\cdot)$ is the zeroth order modified Bessel function of the second kind [9], which for small values of $z$ is a constant ${ }^{1}$. Therefore, for small values of $z$ the first order approximation of $f_{Z}(z)$ is $z^{L}$. This shows that the CDF of $Z, F_{Z}(z)$, has first order approximation equal to $z^{L+1}$, which indicates the diversity order of $L+1$. Now consider the CDF of $W \triangleq$ $Y_{1} \cdot Y_{2} \cdot Y_{3}=Z \cdot Y$, where $Y \sim \chi_{2(N-M+1)}$ :

$$
\begin{aligned}
F_{W}(w) & =\operatorname{Pr}(W \leq w)=\operatorname{Pr}(Z \cdot Y \leq w) \\
& =\int_{0}^{\infty} f_{Z}(z) F_{Y}\left(\frac{w}{z}\right) d z \\
& =\alpha \int_{0}^{\infty} z^{L} K_{0}(2 \sqrt{z}) e^{-\frac{w}{z}} \sum_{k=L+2}^{\infty} \frac{w^{k-1}}{z^{k-1}(k-1) !} d z
\end{aligned}
$$

${ }^{1}$ For small values of $x: K_{m}(x) \sim \frac{\Gamma(m)}{2}(2 / x)^{m} \quad$ [9]. 

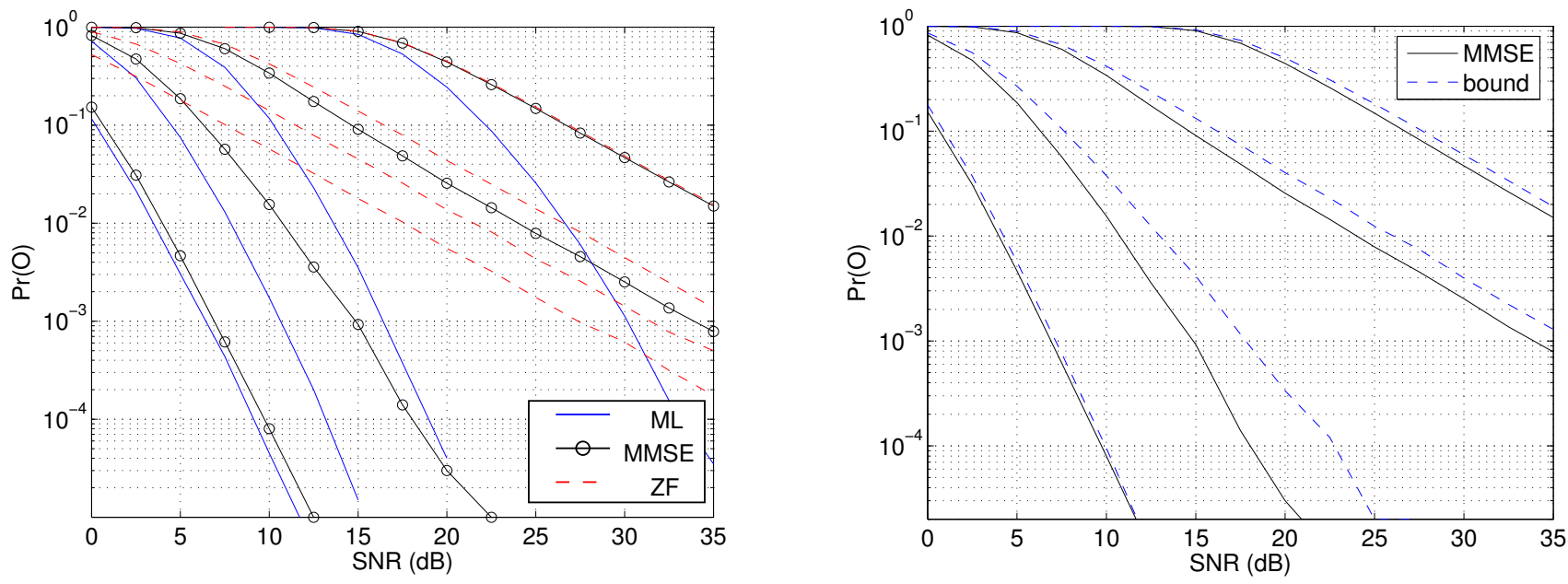

Fig. 2. Left: Comparison of receivers. Right: MMSE outage and the upper bound (15). $M=N=2$ and the curves show rates $\mathrm{R}=1,2,4,10 \mathrm{bits} / \mathrm{sec} / \mathrm{Hz}$.
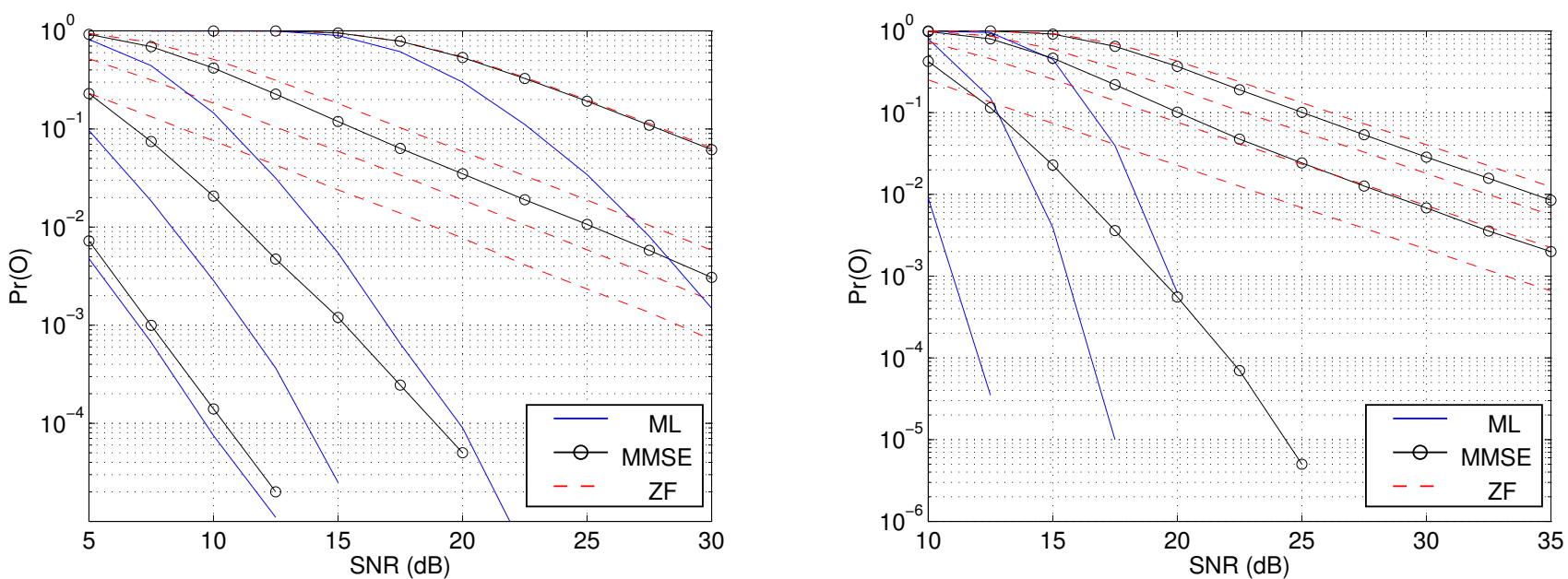

Fig. 3. Comparison of receivers. Left: $M=N=2$, correlated transmit antennas with $\rho_{t}=0.5, \mathrm{R}=1,2,4,10$ bits/sec/Hz. Right: $M=N=4$, uncorrelated, $\mathrm{R}=4,8,12,16 \mathrm{bits} / \mathrm{sec} / \mathrm{Hz}$.

where $\alpha$ is a constant. The first order approximation of above around zero is

$$
w^{L+1} \int_{0}^{\infty} \frac{\alpha}{z(L+1) !} K_{0}(2 \sqrt{z}) d z
$$

Thus, $F_{W}(w)$ behaves like the $(L+1)$ th power of $w$, indicating the diversity order $L+1$. This procedure can be applied recursively to find that the first order approximation of the CDF of $Y_{1} \cdot Y_{2} \cdots Y_{M}$ behaves like $w^{L+1}$. As mentioned, the product term in (20) dominates the diversity. We just showed that the product term $Y_{1} \cdot Y_{2} \cdots Y_{M}$ has the diversity order $L+1$. Therefore, the ZF diversity order is $L+1$.

Proof of Theorem 2: First, we state and prove the following lemma.

Lemma 1: Let

$$
I_{M}=\int_{\sum_{i} \lambda_{i} \leq x} \ldots \int e^{-\sum_{i} \lambda_{i}} \prod_{i=1}^{M} \lambda_{i}^{k_{i}} d \lambda_{1} \cdots d \lambda_{M} .
$$

$I_{M}$ is polynomial in $x$ with the minimum exponent of $g(M)=$
$M+\sum_{i=1}^{M} k_{i}$, where $M$ and $k_{i}$ are integers.

Proof: With some algebra, one can obtain

$$
\begin{aligned}
\int_{0}^{x} \lambda^{m} e^{-\lambda} d \lambda & =m ! \sum_{i=m+1}^{\infty} \frac{x^{i}}{i !} \\
\int_{0}^{x} \lambda^{m}(x-\lambda)^{n} e^{-\lambda} d \lambda= & \sum_{j=0}^{n} C(j, n)(-1)^{j} x^{n-j}(m+j) ! \\
& \times \sum_{\ell=m+j+1}^{\infty} \frac{x^{\ell}}{\ell !}
\end{aligned}
$$

where $m$ and $n$ are integers and $C(\cdot, \cdot)$ are the binomial coefficients. Using (23) and (24), $I_{2}$ can be calculated as

$$
I_{2}=\sum_{i=k_{1}+1}^{\infty} \frac{n !}{i !} \sum_{j=0}^{i} C(j, i)(-1)^{j} x^{i-j}\left(k_{2}+j\right) ! \sum_{\ell=k_{2}+j+1}^{\infty} \frac{x^{\ell}}{\ell !}
$$

where the minimum exponent of $x$ is $g(2)=k_{1}+k_{2}+2$.

Now we use induction. Assume that $I_{M-1}=$ 


$$
\begin{aligned}
& \sum_{i=g(M-1)}^{\infty} a_{i} x^{i} \text {. Then } \\
& I_{M}=\int_{0}^{x} \lambda_{M}^{k_{M}} e^{-\lambda_{M}} \int_{M-1}^{\sum_{i} \lambda_{i} \leq x-\lambda_{M}} e^{-\sum_{i}^{M-1} \lambda_{i}} \prod_{i=1}^{M-1} \lambda_{i}^{k_{i}} d \lambda_{1} \cdots d \lambda_{M} \\
& =\sum_{i=g(M-1)}^{\infty} a_{i} \int_{0}^{x} \lambda_{M}^{k_{M}} e^{-\lambda_{M}}\left(x-\lambda_{M}\right)^{i} d \lambda_{M} \\
& =\sum_{i=g(M-1)}^{\infty} a_{i} \sum_{j=0}^{i} C(j, i)(-1)^{j} x^{i-j}\left(k_{M}+j\right) ! \sum_{\ell=k_{M}+j+1}^{\infty} \frac{x^{\ell}}{\ell !}
\end{aligned}
$$

where (25) is obtained using (24). Equation (25) indicates that $I_{M}$ is polynomial in $x$ with the minimum exponent of $g(M-$ 1) $+k_{M}+1=g(M)$.

To prove Theorem 2, we first note that

$$
\prod_{i<j}\left(\lambda_{i}-\lambda_{j}\right)^{2}=\sum_{\left(k_{1}, \ldots, k_{M}\right) \in \mathcal{S}} p\left(k_{1}, \cdots, k_{M}\right) \prod_{i=1}^{M} \lambda_{i}^{k_{i}},
$$

where the set $\mathcal{S}$ is a subset of the $\left(k_{1}, \cdots, k_{M}\right)$ indexes that $\sum_{i=1}^{M} k_{i}=M(M-1)$, and $p\left(k_{1}, \cdots, k_{M}\right)$ is the corresponding integer coefficient.

In low spectral efficiency, $R<M \log \left(\frac{M}{M-1}\right)$, we can write the right-hand side of (15) as

$$
\begin{aligned}
& K_{M, N} \int_{\mathcal{A}} \ldots \int^{-\sum_{i} \lambda_{i}} \prod_{i=1}^{M} \lambda_{i}^{N-M} \prod_{i<j}\left(\lambda_{i}-\lambda_{j}\right)^{2} d \lambda_{1} \cdots d \lambda_{M} \\
& =K_{M, N} \sum_{\left(k_{1}, \ldots, k_{M}\right) \in \mathcal{S}} p\left(k_{1}, \cdots, k_{M}\right) \\
& \quad \times \int \cdots \int e^{-\sum_{i} \lambda_{i}} \prod_{i=1}^{M} \lambda_{i}^{N-M+k_{i}} d \lambda_{1} \cdots d \lambda_{M} \cdot
\end{aligned}
$$

We now bound the integration region $\mathcal{A}$, from inside and from outside, by polyhedra. Then we use a sandwich argument by showing that the integration over the inner and outer polyhedra gives the same asymptotic performance.

Using a direct extension of the argument developed in the two-dimensional case (proceeding Equation (18)), it can be seen that the polyhedron defined by $\lambda_{i} \geq 0, \sum_{i} \lambda_{i} \leq c_{M}$, where

$$
c_{M}=\rho^{-1} \frac{M\left(1-2^{-\frac{R}{M}}\right)}{1+M\left(2^{-\frac{R}{M}}-1\right)},
$$

contains the integration region $\mathcal{A}$. Therefore an integral over this polyhedron upper bounds the outage probability.

Now consider another polyhedron defined by $\lambda_{i} \geq 0, \sum_{i} \lambda_{i} \leq \tilde{c}_{M}$, where $\tilde{c}_{M}$ is proportional to $\rho^{-1}$ but small enough so that $\mathcal{A}$ contains this polyhedron. The base of this polyhedron can be calculated similarly to Section IV.

$$
\tilde{c}_{M}=\rho^{-1}\left(2^{R / M}-1\right)
$$

Integration over this new polyhedron, which is characterized by $\tilde{c}_{M}$, lower bounds $\operatorname{Pr}(\mathcal{A})$.
Finally Lemma 1 establishes that the asymptotic behavior of (27), while integrating over either of the two polyhedra, is the same. Each multiple integral in (27) is in the form of $I_{M}$ of Lemma 1, i.e., polynomial in $c_{M}$ with smallest exponent $M+\sum_{i=1}^{M}\left(N-M+k_{i}\right)=M N$. Therefore, the upper bound (15) decays with $\rho^{-M N}$ in low spectral efficiency, indicating diversity order is no less than $M N$. At the same time, $M N$ is actually the maximum possible diversity order, so the outage probability of MMSE receiver has diversity of $M N$.

We now proceed to show the high-rate result, where the developments parallel those for $M=2$ in (19). For $R>$ $M \log M$ the outage region $\mathcal{A}$ can be upper and lower bounded with orthogonal slabs along the coordinates. The first set that is a subset of $\mathcal{A}$ has $M$ orthogonal slabs where the $j$-th slab is defined as $\lambda_{j} \leq d_{M}$ and $\lambda_{i \neq j} \geq 0$, where $d_{M}=\rho^{-1}\left(\frac{1}{M} 2^{\frac{R}{M}}-1\right)$. The outage region $\mathcal{A}$ is a subset of the second set of slabs whose definition is the same as the first set with $d_{M}$ replaced with $\tilde{d}_{M}$, which is also proportional to $\rho^{-1}$ and $\tilde{d}_{M}>d_{M}$.

Therefore, the right-hand side of the bound (15) is the same as (27) with the exception that the integration region $\mathcal{A}$ could be either of above sets. Considering the possibility of some zero $k_{i}$ in (26) and the unbounded shape of $\mathcal{A}$, there are dominating terms such as

$$
\int_{\lambda_{j} \leq d_{M}, \lambda_{i \neq j} \geq 0} e^{-\sum_{i} \lambda_{i}} \lambda_{j}^{N-M} \prod_{i \neq j}^{M} \lambda_{i}^{N-M+k_{i}} d \lambda_{1} \cdots d \lambda_{M},
$$

which is polynomial in $d_{M}$ with the minimum exponent of $N-M+1$. This indicates that the bound (15) decays with $\rho^{-(N-M+1)}$ in high spectral efficiency. This completes the proof of Theorem 2 .

\section{REFERENCES}

[1] G. Foschini, G. Golden, R. Valenzuela, and P. Wolniansky, "Simplified processing for high spectral efficiency wireless communication employing multi-element arrays," Journal on Selected Areas in Communications, vol. 17, pp. 1841-1852, Nov. 1999.

[2] N. Prasad and M. K. Varanasi, "Outage analysis and optimization for multiaccess and V-BLAST architecture over MIMO Rayleigh fading channels," in Proc. of 41th Annual Allerton Conference on Communication, Control, and Computing, 2003.

[3] T. Guess, H. Zhang, and T. V. Kotchiev, "The outage capacity of BLAST for MIMO channels," in Proc. IEEE ICC, May 2003, pp. 2628-2632.

[4] J. H. Winters, J. Salz, and R. D. Gitlin, "The impact of antenna diversity on the capacity of wireless communication systems," IEEE Transactions on Communications, vol. 43, pp. 1740-1750, February/March/April 1994.

[5] M. Rupp, C. Mecklenbrauker, and G. Gritsch, "High diversity with simple space time block-codes and linear receivers," in Proc. IEEE GLOBECOM, San Francisco, CA, November 2003, pp. 302-306.

[6] E. K. Onggosanusi, A. G. Dabak, T. Schmidl, and T. Muharemovic, "Capacity analysis of frequency-selective MIMO channels with suboptimal detectors," in Proc. IEEE ICASSP, 2002, pp. 2369-2372.

[7] H. Gao, P. J. Smith, and M. V. Clark, "Theoretical reliability of MMSE linear diversity combining in Rayleigh-fading additive interference channels," IEEE Transactions on Communications, vol. 46, pp. 666-672, May 1998.

[8] L. Zheng and D. Tse, "Diversity and multiplexing: A fundamental tradeoff in multiple-antenna channels," IEEE Transactions on Information Theory, vol. 49, no. 5, pp. 1073-1096, May 2003.

[9] I. S. Gradshteyn and I. M. Ryzhik, Table of Integrals, Series, and Products, San Diego, CA: Academic Press, 5th edition, 1994. 